\title{
Effects of Long-Term Oral Administration of Ketoprofen in Clinically Healthy Beagle Dogs
}

\author{
Tatsuya NARITA ${ }^{1,2)}$, Nobuyuki TOMIZAWA2), Reeko SATO ${ }^{3)}$, Masanobu GORYO ${ }^{4)}$ and Shigeo HARA ${ }^{2)}$ \\ ${ }^{1)}$ Department of Clinical Veterinary Science, The United Graduate School of Veterinary Sciences, Gifu University, Gifu 501-1193 and \\ ${ }^{2)}$ Departments of Veterinary Surgery, ${ }^{3)}$ Veterinary Internal Medicine and ${ }^{4)}$ Veterinary Pathology, Faculty of Agriculture, Iwate \\ University, Morioka 020-8550, Japan
}

(Received 2 August 2004/Accepted 9 May 2005)

\begin{abstract}
To investigate the adverse effects of long-term administration of ketoprofen in dogs, ketoprofen (1 mg/kg) was administered to five clinically healthy beagle dogs (ketoprofen group) and gelatin capsules (control group) were administered to four clinically healthy beagle dogs for 30 days. We monitored the dogs through periodic physical examination, blood analyses, endoscopic examinations, fecal occult blood tests, renal function tests, urinalysis, urinary enzyme indices and cuticle bleeding time analysis. The lesions in the stomach, especially in the pyloric antrum, and fecal occult blood progressively worsened in the ketoprofen group. However, the differences between the ketoprofen group and the control group were not statistically significant. One dog in the ketoprofen group temporarily exhibited a decrease in renal plasma flow and two dogs exhibited enzymuria. However, these changes did not persist and the other examinations showed no significant difference between premedication and postmedication in the ketoprofen group. Therefore, the adverse effects of long-term administration of ketoprofen observed in this study were not clinically important in healthy dogs. Nevertheless, further investigation of adverse renal effects from long-term administration of ketoprofen is necessary in the dogs with subclinical renal disease. KEY WORDS: adverse effect, canine, Ketoprofen, long-term administration.
\end{abstract}

J. Vet. Med. Sci. 67(9): 847-853, 2005

Non-steroidal anti-inflammatory drugs (NSAIDs) are frequently used for the treatment of musculoskeletal disease in veterinary medicine, and are generally recognized for their analgesic, antipyretic, and anti-inflammatory properties [18, 20]. These effects are in general mediated by the inhibition of inflammatory cyclooxygenase (COX-2). COX-2 is activated in injured tissue and produces prostaglandins (PGs) for hyperalgesia by sensitizing sensory nerve endings to various other mediators such as bradykinin, histamine and substance P. However, most NSAIDs also have various adverse effects including gastrointestinal irritation in dogs $[4,5,35]$, renal disorders in dogs and rats [7, 25, 34], and inhibition of platelet aggregation in dogs $[15,17]$ caused by the suppression of homeostatic cyclooxygenase (COX-1), which produces PGs for physiological roles within these tissues and cells [30]. Hepatotoxicity has also been reported after the administration of some NSAIDs in dogs [2, 21, 23].

Musculoskeletal diseases such as osteoarthritis and intervertebral disk disease, that are commonly a problem in geriatric dogs, produce chronic pain. Therefore, long-term administration of NSAIDs is necessary in order to reduce pain and improve the quality of life. However, NSAIDs are often prescribed only for short-term use in veterinary cases because of an anticipated increase in the incidence of side effects with prolonged use. There is a little information regarding administration for longer than seven days in veterinary medicine.

Ketoprofen, a new NSAID derived from propionic acid, has potent analgesic, antipyretic and anti-inflammatory effects in dogs and cats $[6,13,31,36]$. Ketoprofen is a potent inhibitor of COX that exhibits some inhibitory effects on lipoxygenase and the synthesis of bradykinin in vitro.
Therefore, it inhibits the synthesis and release of PGs, and to some extent, synthesis of leukotrienes, leading to the peripheral analgesic effect common to most NSAIDs. Ketoprofen also provides analgesic effects at the central level. Forsyth et al. [10] reported that ketoprofen was less damaging to the gastroduodenal mucosa than aspirin, when those drugs were given over seven days in dogs. However, their report estimated only adverse gastrointestinal effects. Little information exists about other adverse effects of ketoprofen, such as on renal function and platelet aggregation, and no observations of ketoprofen administration for longer than seven days have been reported.

Aging is correlated with increased incidence of organ dysfunction including renal disease, which may result from a decrease in homeostatic COX. Therefore, it is also important to investigate the effects of NSAIDs on renal function and platelet aggregation, as well as on the gastrointestinal mucosa, and to ensure the safety of long-term administration of NSAIDs in geriatric animals having low levels of homeostatic COX. The purpose of the present study was to investigate the adverse effects of long-term administration of ketoprofen on the gastrointestinal tract, kidneys and platelets in healthy beagle dogs.

\section{MATERIALS AND METHODS}

Dogs: Nine clinically healthy beagle dogs (3 intact males and 6 intact females) were used in the present study. Dogs ranged from 6 to 35 months of age (mean \pm SD; $14.8 \pm 9.4$ months) and weighed between 8 and $14.8 \mathrm{~kg}$ (mean $\pm \mathrm{SD}$; $9.85 \pm 2 \mathrm{~kg})$.

Experimental design: The dogs were randomly assigned 
to two treatment groups. The dogs of the ketoprofen group (5 females, body weight $9.7 \pm 0.8 \mathrm{~kg}$, No. 1-5) orally received ketoprofen $(1 \mathrm{mg} / \mathrm{kg}$ once a day: Ketofen, Merial Japan Co., Ltd., Fukushima, Japan), and the dogs of the control group ( 3 males and 1 female, body weight $10.1 \pm 3.2 \mathrm{~kg}$, No. 6-9) orally received a gelatin capsule (empty gelatin capsules, Kobayashi Capsulae Inc., Hyogo, Japan) once a day. Treatments were carried out for thirty days and the drugs were given at 8:00-9:00 a.m. All experimental dogs were housed in cages and fed a commercial dog food (Eukanuba Original, Iams Japan Inc., Tokyo, Japan) once a day after drug administration in the morning. Water was given ad libitum. Physical examinations, including observation for anorexia, vomiting, diarrhea, signs of depression and abdominal pain were performed in both groups once a day before and every day after treatments were started. Venous blood analyses including CBC and serum biochemical analysis were performed by an automatic analyzer (Hitachi Automatic Analyzer 7060, Hitachi Inc., Tokyo, Japan) in the ketoprofen group before and every 10 days after starting the treatment. This study was approved by the Animal Research Committee of Iwate University.

Endoscopic examination: Endoscopic examination of the gastrointesitinal mucosa was performed in both groups before, every 7 days after starting the treatment, and 3 days after treatment was stopped, just before necropsy. Food was withheld for $24 \mathrm{hr}$ and water was withheld for $6 \mathrm{hr}$ before anaesthesia. Anaesthesia was induced with thiopental sodium (15 to $25 \mathrm{mg} / \mathrm{kg}$, IV: Ravonal, Tanabe Pharmaceutical Co., Ltd., Osaka, Japan), and an intratracheal tube was intubated. Anaesthesia was maintained with isoflurane (Forane, Dainabot Co., Ltd., Tokyo, Japan) in oxygen for the duration of the endoscopic examination. The gastrointestinal tract was examined using a videoendoscope (Olympus ves, Olympus Avs Co., Ltd., Tokyo, Japan), and the images of the mucosa were photographed. The number and size of the mucosal lesions were recorded and graded according to the scale reported by Forysh et al. [9, 10]. Briefly, the mucosal lesions are graded from 0 to 6 . In this grading scale, grade 0 means that there is no lesion, and grades 1 to 4 mean that there are 1 to 5 (grade1), 6 to 15 (grade 2), 16 to 25 (grade 3 ) or more than 25 (grade 4) punctate erosions and/or hemorrhages, respectively. Further- more, there are 1 to 5 invasive erosions in grade 4, and more than 5 invasive erosions in grade 5. Grade 6 means that there are ulcers. With these criteria, invasive erosions were defined as extensive hemorrhages or erosions with evidence of invasion as indicated by detectable depth/breadth significantly greater than a pinhead-size discontinuation of the mucosal epithelium. An ulcer was defined as a lesion producing a wide discontinuation of the mucosa and having a crater-like center.

Fecal occult blood tests: A test for fecal occult blood reaction in fresh feces was performed in both groups before and after 6, 13, 20, 27 days of the treatment. A commercially available kit (Occult Blood Slide Shionogi II, Shionogi Co., Ltd., Osaka, Japan) that uses the tetramethylbenzidine and guaiac method was used in the present study. Analysis was performed and graded according the manufacturer's instructions (Table 1).

Renal function tests: Renal function was assessed by determination of renal plasma flow (RPF) and glomerular filtration rate (GFR) in the ketoprofen group before and every 10 days after starting the treatment. Para-aminohippurate sodium (PAH: Daiichi Pharmaceutical Co., Ltd., Tokyo, Japan) clearance $\left(\mathrm{C}_{\mathrm{PAH}}\right)$ and endogenous creatinine clearance $\left(\mathrm{C}_{\mathrm{CRE}}\right)$ were measured to assess RPF and GFR, respectively. $\mathrm{C}_{\mathrm{PAH}}$ and $\mathrm{C}_{\mathrm{CRE}}$ were analyzed simultaneously. CPAH was analyzed by bolus administration of PAH (40 $\mathrm{mg} / \mathrm{kg}, \mathrm{IV})$. Blood was collected at $30 \mathrm{~min}$ before PAH administration, and 35 and 65 min after PAH administration. Urine was collected from 35 to $75 \mathrm{~min}$ after $\mathrm{PAH}$ administration. $\mathrm{C}_{\mathrm{PAH}}$ and $\mathrm{C}_{\mathrm{CRE}}$ were estimated using the formulae below.

$$
\begin{aligned}
& \mathrm{C}_{\text {PAH }}(\mathrm{m} l / \mathrm{min} / \mathrm{kg})=\text { Urine }_{\text {PAH }}(\mathrm{mg} / \mathrm{d} l) \times \text { Volume }(\mathrm{m} l / \mathrm{min} / \\
& \mathrm{kg}) / \text { Plasma }_{\text {PAH }}(\mathrm{mg} / \mathrm{d} l) \\
& \mathrm{C}_{\text {Cre }}(\mathrm{m} l / \mathrm{min} / \mathrm{kg})=\text { Urine }_{\text {Cre }}(\mathrm{mg} / \mathrm{d} l) \times \text { Volume }(\mathrm{m} l / \mathrm{min} / \\
& \mathrm{kg}) / \text { Plasma } \\
& \text { Cre }
\end{aligned}
$$

Urinalysis and urinary enzyme activities: Urinalysis, including specific gravity, dipstick analysis and sediment examination was performed in the ketoprofen group before and every 5 days after starting treatment. In addition, urinary enzyme activities, including $\mathrm{N}$-acetyl- $\beta$-D-glucosaminidase (NAG) and $\gamma$-glutamyl transpeptidase (GGT),

Table 1. Fecal occult blood grading scale used in the present study

\begin{tabular}{rll}
\hline Grade & Evaluation & Criteria $^{\mathrm{a}, \mathrm{b})}$ \\
\hline 0 & Negative & No change in the smeared surface \\
1 & Weakly positive & The smeared surface seems slightly blue green \\
2 & Positive & The smeared surface turns blue green \\
3 & Moderately positive & The smeared surface turns definitely blue green \\
4 & Strongly positive & $\begin{array}{l}\text { The smeared surface becomes dark blue immediately } \\
\text { after the color reagent is applied }\end{array}$ \\
& &
\end{tabular}

a) Evaluation of result is performed after $30 \mathrm{sec}$ from applying the color reagent.

b) Determination of fecal occult blood is performed by both the tetramethylbenzidine method and guaiac method simultaneously over grade 1. 
were measured in the ketoprofen group before and every 3 days after starting treatment. Urinalysis was performed using a urine dipstick (Aution Sticks 5EA, Arkray Inc., Kyoto, Japan) and the specific gravity of urine was assayed using a refractometer (Clinical Refractometer T2-NE, Atago Co., Ltd., Tokyo, Japan). Microscopic evaluation of urine was performed on urine sediment stained with SternheimerMalbin stain (URI-CEL, Cambridge Diagnostic Products Inc., Fort Lauderdale, Florida, U.S.A.). Urine for the measurement of NAG activity, GGT activity and creatinine concentration was collected aseptically by a sterilized silicone elastomer coated Foley catheter (Ruschi Ultrasil, Rushi Asia Pacific Sdn Bhd., Tokyo, Japan) and centrifuged at $500 \times \mathrm{g}$ for $15 \mathrm{~min}$ to obtain the supernatant. The samples for NAG activity and creatinine concentration were immediately stored at $-20^{\circ} \mathrm{C}$ and samples for GGT activity were immediately stored at $4^{\circ} \mathrm{C}$. Measurements were performed within a week of storage. Urinary NAG activity was determined colorimetrically on a spectrophotometer (Spectrometer 10550, Hitachi Inc., Tokyo, Japan) at $405 \mathrm{~nm}$ and at $37^{\circ} \mathrm{C}$ using p-nitrophenyl $\mathrm{N}$-acetyl- $\beta$-D-glucosaminide (PNP: PNPNAG, Sigma Chemical Co., St Louis, MO, U.S.A.) as a substrate [33]. Urinary GGT activity was measured by an automatic analyzer (Hitachi Automatic Analyzer 7060, Hitachi Inc., Tokyo, Japan). Urinary creatinine concentration was measured by the modified Folin method [16]. Urinary NAG and GGT indices were calculated by the formulae below.

NAG index $(\mathrm{U} / \mathrm{g})=$ urinary NAG activity $(\mathrm{U} / l) /$ urinary creatinine concentration $(\mathrm{g} / \mathrm{l})$

GGT index $(\mathrm{U} / \mathrm{g})=$ urinary GGT activity $(\mathrm{U} / l) /$ urinary creatinine concentration $(\mathrm{g} / l)$

Cuticle bleeding time: Cuticle bleeding time (CBT) was measured as the primary haemostatic function test in the ketoprofen group before and every 7 days after starting treatment. Cuticle bleeding time (CBT) was measured based on the method described by Giles et al. [11] after endoscopic examination.

Necropsy: All dogs except for No. 9 were euthanized for necropsy on day 33 by the administration of pentobarbital sodium (Nembutal, Dainippon Pharmaceutical Co., Ltd., Osaka, Japan). The stomach, duodenum, jejunum, ileum, cecum, colon, kidneys, liver, and bone marrow in each dog were examined for gross lesions, and each sample was fixed in buffered 10\% formalin (Mildform, Toyo Science Co., Ltd., Tokyo, Japan). After fixation, each sample was embedded in paraffin, sectioned at $3 \mu \mathrm{m}$, and stained with H\&E. All specimens and tissues were examined by a pathologist who was blinded to the treatment status of each dog.

Statistical analysis: Results of CBC, blood biochemistry, RPF, GFR and CBT were analyzed using two-factor ANOVA, and endoscopic score and fecal occult blood grade were analyzed using Mann-Whitney's $U$ test. All comparisons were performed by the use of a computerized statistical program, and a probability of less than 0.05 was considered significant for all comparisons.

\section{RESULTS}

Physical examinations, hematologic examinations and serum biochemical analyses: The physical examinations revealed no abnormalities in either group after treatment was started. Clinical signs of gastrointestinal disorders, such as anorexia, vomiting, diarrhea and abdominal pain, were not observed. Furthermore, no significant differences were observed between the pre- and post-treatment blood parameters in either group.

Endoscopic examination: No lesions were observed in the esophagus of any dog during the experimental period. Over the course of the study, all dogs in the ketoprofen group and two dogs in the control group developed small mucosal erosions and haemorrhage in the stomach. Two dogs (No. 4 \& No. 5) in the ketoprofen group developed invasive erosions in the stomach and one dog in the control group developed a small mucosal erosion in the proximal duodenum. In both groups, especially in the ketoprofen group, mucosal lesions of the pyrolic antrum were more severe than those in the other portions. However, no significant differences were observed in the gastroduodenal lesion scores between the ketoprofen group and the control group. In addition, none of the dogs in either group had lesions on days 33 (Table 2).

Figure 1 presents the total gastroduodenal lesion scores (mean $\pm \mathrm{SD}$ ) for the two treatment groups. Dogs receiving ketoprofen had higher scores $(1.8 \pm 2.5,3.0 \pm 2.5,3.8 \pm 4.4$, $4.0 \pm 4.8$ ) on days $7,14,21$ and 28 , respectively, than dogs receiving gelatin capsules $(0.8 \pm 1.5,1.8 \pm 2.1,1.5 \pm 1.7,2.3$ \pm 2.6 ). However, no significant differences were observed in the total gastroduodenal lesion scores between the ketoprofen group and the control group.

Fecal occult blood tests: Positive results were observed by both methods in the ketoprofen group on days 13 to 27 and in the control group on day 27. Both methods demonstrated an increase in grade over time, especially in the ketoprofen group (Fig. 2). However, no significant differences were observed between the ketoprofen group and the control group.

Renal function tests: The reference ranges for RPF and GFR, determined by the preliminary study, were $12.7 \pm 2.3$ $\mathrm{m} l / \mathrm{min} / \mathrm{kg}$ and $4.5 \pm 1.0 \mathrm{ml} / \mathrm{min} / \mathrm{kg}$ (mean $\pm \mathrm{SD}$ ), respectively. No significant differences were observed in RPF between pre-treatment and post-treatment. However, the RPF of dog No. 5 (ketoprofen group) was below the reference range at 20 and 30 days $(8.1 \mathrm{ml} / \mathrm{min} / \mathrm{kg}$ and $9.4 \mathrm{ml} / \mathrm{min} /$ $\mathrm{kg}$, respectively). Significant differences were also not observed in GFR between pre-treatment and post-treatment (Fig. 3).

Urinalysis and urinary enzyme activities: The dipstick test showed few changes over the sampling period except for dog No. 5, which exhibited mild to moderate proteinuria at days 5, 10, 15, 20 and 30, and which correlated with the number of renal tubular epithelial (RTE) cells in the urine ( 2 to 3 RTE cells / HPF were observed at 5 to 15 days). The 
Table 2. Mean score of gastrointestinal lesions of dogs at pretreatment and during the experimental period (day 7 to 33 ) in the ketoprofen group and the control group

\begin{tabular}{|c|c|c|c|c|c|c|c|}
\hline & Group & Pretreatment & Day 7 & Day 14 & Day 21 & Day 28 & Day 33 \\
\hline \multirow{2}{*}{ Esophagus } & Ketoprofen & $0(0)$ & $0(0)$ & $0(0)$ & $0(0)$ & $0(0)$ & $0(0)$ \\
\hline & Control & $0(0)$ & $0(0)$ & $0(0)$ & $0(0)$ & $0(0)$ & $0(0)$ \\
\hline \multirow{2}{*}{ Cardia/fundus } & Ketoprofen & $0(0)$ & $0(0)$ & $0(0)$ & $0.2(0$ to 1$)$ & $0.8(0$ to 3$)$ & $0(0)$ \\
\hline & Control & $0(0)$ & $0(0)$ & 0.5 (0 to 2$)$ & 0.25 (0 to 1$)$ & $0.75(0$ to 2$)$ & $0(0)$ \\
\hline \multirow{2}{*}{ Stomach body } & Ketoprofen & $0(0)$ & $0.2(0$ to 1$)$ & $0.8(0$ to 2$)$ & $0.2(0$ to 1$)$ & $0.2(0$ to 1$)$ & $0(0)$ \\
\hline & Control & $0(0)$ & $0(0)$ & $0(0)$ & $0(0)$ & $0(0)$ & $0(0)$ \\
\hline \multirow{2}{*}{ Body-antrum junction } & Ketoprofen & $0(0)$ & $0.2(0$ to 1$)$ & $0.2(0$ to 1$)$ & $0.8(0$ to 4$)$ & $0(0)$ & $0(0)$ \\
\hline & Control & $0(0)$ & $0(0)$ & 0.25 (0 to 1$)$ & $0(0)$ & $0(0)$ & $0(0)$ \\
\hline \multirow{2}{*}{ Angular ventriculi } & Ketoprofen & $0(0)$ & $0(0)$ & $0(0)$ & $0.2(0$ to 1$)$ & $0.2(0$ to 1$)$ & $0(0)$ \\
\hline & Control & $0(0)$ & $0(0)$ & $0(0)$ & $0(0)$ & $0(0)$ & $0(0)$ \\
\hline \multirow{2}{*}{ Pyrolic antrum } & Ketoprofen & $0(0)$ & 0.8 (0 to 2$)$ & $1.4(0$ to 3$)$ & $2(0$ to 5$)$ & $2.4(0$ to 5$)$ & $0(0)$ \\
\hline & Control & $0(0)$ & $0.5(0$ to 2$)$ & $0.25(0$ to 1$)$ & 0.75 (0 to 2$)$ & $1(0$ to 2$)$ & $0(0)$ \\
\hline \multirow{2}{*}{ Pyrolus } & Ketoprofen & $0(0)$ & $0.6(0$ to 2$)$ & $0.6(0$ to 2$)$ & $0.4(0$ to 1$)$ & $0.4(0$ to 1$)$ & $0(0)$ \\
\hline & Control & $0(0)$ & 0.25 (0 to 1$)$ & $0.5(0$ to 1$)$ & 0.5 (0 to 1$)$ & 0.5 (0 to 1$)$ & $0(0)$ \\
\hline \multirow{2}{*}{ Duodenum } & Ketoprofen & $0(0)$ & $0(0)$ & $0(0)$ & $0(0)$ & $0(0)$ & $0(0)$ \\
\hline & Control & $0(0)$ & $0(0)$ & 0.25 (0 to 1$)$ & $0(0)$ & $0(0)$ & $0(0)$ \\
\hline
\end{tabular}

( ): Range scale.

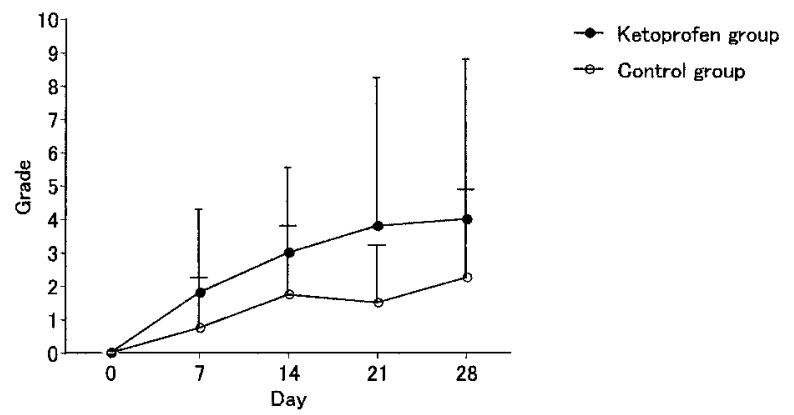

Fig. 1. Total lesion scores determined by gastrointestinal endoscopy before treatment (day 0) and during the experimental period (day 7 to 28) in the ketoprofen group and the control group. Results represent mean $\pm \mathrm{SD}$.

reference range for the NAG index is $3.2 \pm 2.4 \mathrm{U} / \mathrm{g}$, as reported by Sato et al. [28], and the reference range for the GGT index is $31.6 \pm 10.4 \mathrm{U} / \mathrm{g}$, as determined by a preliminary study. No marked changes were observed in urinary enzyme indices during the experimental period in the ketoprofen group except for dogs No. 4 and No. 5. The NAG index increased remarkably between days 6 and 18 in dog No. 5, and the GGT index increased mildly at days 6,9 , and 18 in dog No. 5 and at day 30 in dog No. 4 (Fig. 4).
Cuticle bleeding time: All CBT in the ketoprofen group fell within the reference range ( 2 to $8 \mathrm{~min}$, reported by Giles et al. [11]), and no significant difference was observed in CBT during the experimental period (Fig. 5).

Necropsy: Gross lesions were not observed in the stomach, duodenum, jejunum, ileum, cecum, colon, kidney, liver, or bone marrow of any dog. Histopathologically, two dogs (No. 4 and No. 5) in the ketoprofen group exhibited mild lymphoid cell infiltration in the renal medulla, and one $\operatorname{dog}$ (No. 5) in the ketoprofen group exhibited mild depositon of lipofuscin, lymphoid cell infiltration, and multiple siderolic foci in the liver. All other specimens, including gastrointestinal mucosa, were normal.

\section{DISCUSSION}

NSAIDs, including ketoprofen, have been traditionally administered to small animals with chronic pain conditions, for example, degenerative joint disease and cancer, in an effort to relieve pain and improve quality of life. However, NSAIDs may induce gastrointestinal injury $[4,5,35]$, renal failure [7, 25, 34], and inhibit platelet aggregation [15, 17] by several mechanisms. These side effects are correlated with the length of administration and dose of medication. Some NSAIDs may also idiosyncratically induce hepatocellular toxicosis [2, 21, 23]. 


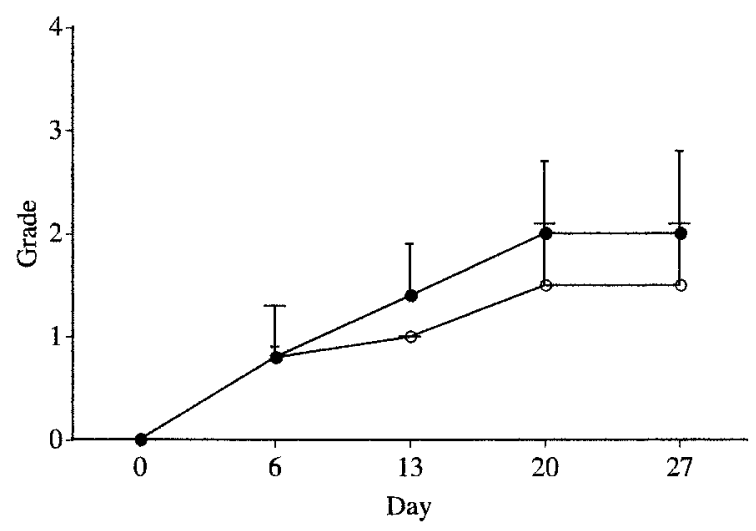

Tetramethylbenzidine method

Ketoprofen group

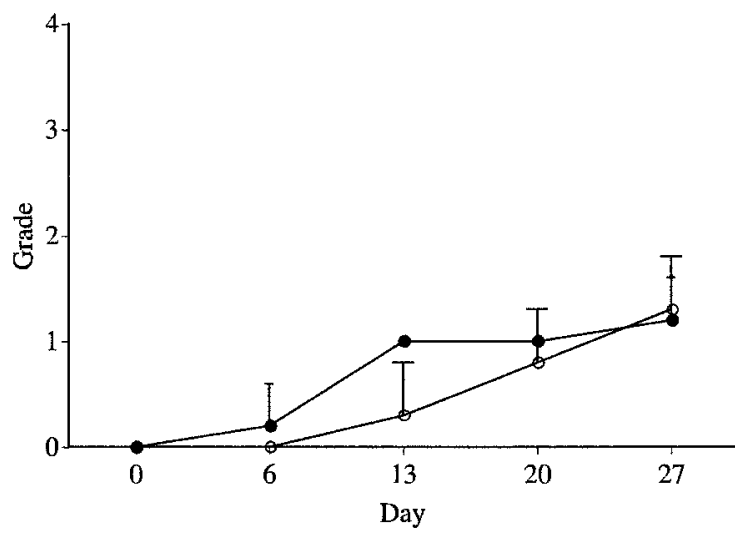

Guaiac method Ketoprofen group

$\rightarrow$ Control group

Fig. 2. Fecal occult blood scores before treatment (day 0) and during the experimental period (day 6 to 27 ) in the ketoprofen group and the control group. Results represent mean $\pm \mathrm{SD}$.

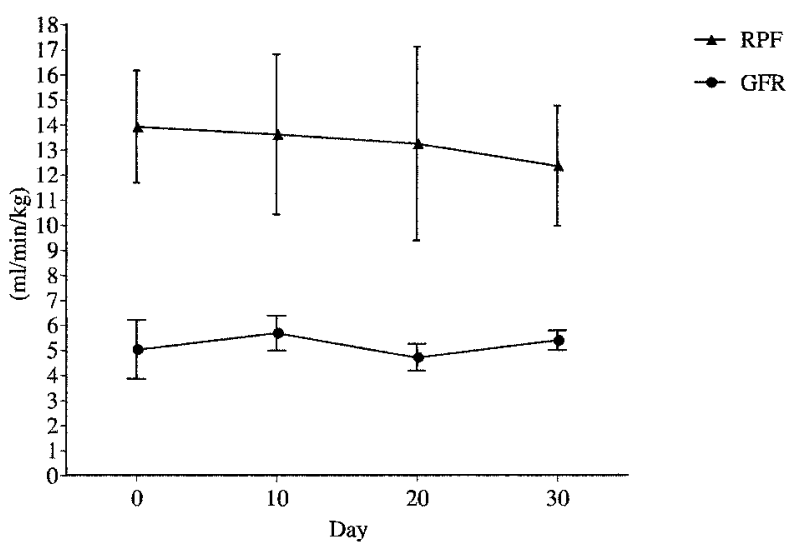

Fig. 3. Changes in renal plasma flow (RPF) and glomerular filtration rate (GFR) in dogs before treatment (day 0 ) and during the experimental period (day 10 to 30 ) in the ketoprofen group. Results represent mean $\pm \mathrm{SD}$.

Gastroenteropathy is the most common side effect caused by the chronic administration of NSAIDs $[9,10]$. In the present study, the pyrolic antrum was the most severely affected site in both groups, especially in the ketoprofen group, and the dogs receiving ketoprofen exhibited more severe lesions than control dogs, although the difference was not statistically significant. These results are similar to the findings of other studies in which gastric mucosa were evaluated after ketoprofen was administered to healthy dogs for 28 days [9, 10]. However, arthritic patients have been reported to be at greater risk of developing NSAID-associated gastropathy than other NSAID users [24], and thus the side effects of long-term administration of ketoprofen in arthritic dogs will have to be investigated in the future.

Fecal occult blood tests were positive in both groups after administration of ketoprofen or gelatin and correlated with the endoscopic examination score in the stomach. The ketoprofen group grade was higher than that of the control group, which indicates that fecal occult blood tests may be useful for detection of gastrointestinal bleeding disorders in dogs treated with NSAIDs.

Adverse renal effects of NSAIDs result primarily from the decreased synthesis of renal vasodilative PGs (PGE2 and PGI2) $[1,3,19,27]$. A previous study of ketoprofenassociated adverse renal effects found decreased endogenous creatinine clearance in dogs receiving ketoprofen before castration surgery [8]. Likewise, a separate study [22] reported decreased $\mathrm{FC}_{\mathrm{Na}}$ and urine specific gravity, and increased renal tubular epithelial cells in urine sediment after ovariohysterectomies in dogs receiving ketoprofen before surgery. Even short-term administration of ketoprofen may carry the risk of adverse renal effects under inhalation anesthesia. However, renal function and chronic renal injury have not been previously investigated under longterm ketoprofen administration. In the present study, urinary NAG and GGT indices were measured as markers of renal injury. These indices are used as markers of various renal disorders $[12,14,26,28,29,33]$. During the experimental period, these urinary enzyme indices were elevated in 2 of 5 dogs. One of these dogs exhibited a temporary decrease in RPF. These results indicate that the urinary enzyme index may be more suitable as a screening test of renal side effects associated with NSAID administration than the measurement of serum BUN and creatinine. However, urinary NAG and GGT indices returned to the reference range at days 21 to 30 despite the continued drug administration. A previous study [32] reported similar effects in rats in response to gentamicin, in which there was a tendency for urinary excretion of NAG to increase transiently. In the present study, histopathological kidney 

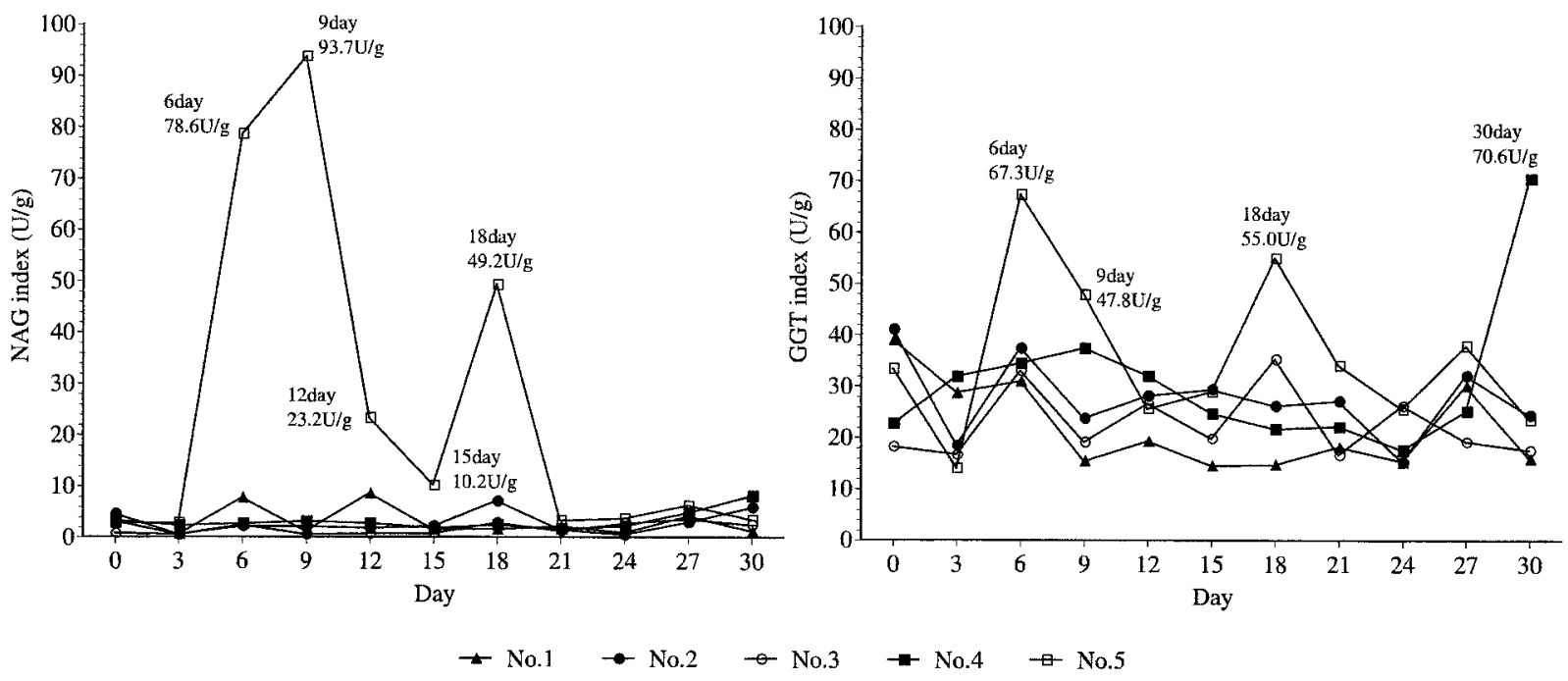

Fig. 4. Changes in NAG index and GGT index in each dog before treatment (day 0) and during the experimental period (day 3 to 30 ) in the ketoprofen group.

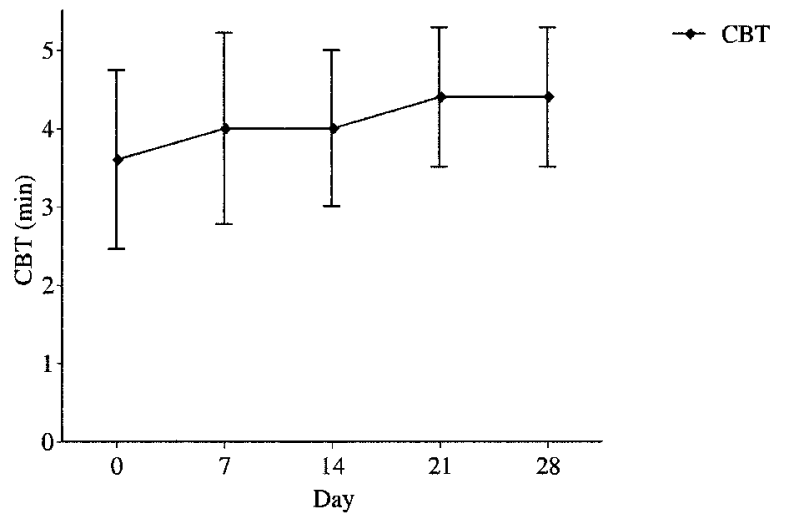

Fig. 5. Changes in cuticle bleeding time in dogs before treatment (day 0 ) and during the experimental period (day 7 to 28 ) in the ketoprofen group. Results represent mean $\pm \mathrm{SD}$.

lesions that had been observed in dogs No. 4 and No. 5 during the experiment, were not observed after the experiment. These findings support the hypothesis that renal injury recovered and surviving nephrons compensated for renal function. Therefore, attentive monitoring of renal function is necessary for dogs with subclinical renal disease, which receive long-term administration of ketoprofen.

Cuticle bleeding time was not significantly longer in the ketoprofen group during the experiment and these dogs did not exhibit either petechia or ecchymosis. These results suggest that bleeding time is not affected in clinically healthy dogs that are administered ketoprofen for 30 days. Upon necropsy, we observed no gross lesions or severe histological lesions, and endoscopic examination performed just before necropsy revealed no lesions in the stomach or duodenum. Thus, our results indicate that the adverse effects of ketoprofen on the digestive tract may diminish within 3 days after administration.

In conclusion, we have reported two important findings in the present investigation. First, adverse gastrointestinal effects tended to occur in animals administered ketoprofen over a long period. However, gastrointestinal lesions healed after administration ceased and thus they were not clinically important in healthy dogs. Secondly, RPF was observed in one out of five healthy dogs and enzymuria was observed in two dogs receiving long-term administration of ketoprofen. Therefore, further investigation of the adverse renal effects of long-term ketoprofen administration appears to be necessary in dogs with subclinical renal disease.

\section{REFERENCES}

1. Black, S. C., Brideau, C., Cirino, M., Belley, M., Bosquet, J., Chan, C. and Rodger, I. W. 1998. Differential effect of a selective cyclooxygenase-2 inhibitor versus indomethacin on renal blood flow in conscious volume-depleted dogs. J. Cardiovasc. Pharmacol. 32: 686-694.

2. Boelsterli, U. A., Zimmerman, H. J. and Kretz-Rommel, A. 1995. Idiosyncratic liver toxicity of nonsteroidal anti-inflammatory drugs: molecular mechanisms and pathology. Crit. Rev. Toxicol. 25: 207-235.

3. Clive, D. M. and Stoff, J. S. 1984. Renal syndromes associated with nonsteroidal anti-inflammatory drugs. New Engl. J. Med. 310: $563-572$.

4. Daehler, M. H. 1986. Transmural pyloric perforation associated with naproxen administration in a dog. J. Am. Vet. Med. Assoc. 189: 694-695.

5. Dow, S. W., Rosychuk, R. A. W., McChesney, A. E. and Curtis, C. R. 1990. Effects of flunixin and flunixin plus prednisone on gastrointestinal tract of dogs. Am. J. Vet. Res. 51: 11311138.

6. Elhakim, M. 1991. A comparison of intravenous ketoprofen with pethidine for postoperative pain relief following nasal surgery. Acta Anaesthesiol. Scand. 35: 279-282. 
7. Elwood, C., Boswood, A., Simpson, K. and Carmichael, S. 1992. Renal failure after flunixin meglumine administration. Vet. Rec. 130: 582-583.

8. Forsyth, S. F., Guilford, W. G. and Pfeiffer, D. U. 2000. Effect of NSAID administration on creatinine clearance in healthy dogs undergoing anaesthesia and surgery. J. Small Anim. Pract. 41: 547-550.

9. Forsyth, S. F., Guilford, W. G., Haslett, S. J. and Godfrey, J. 1998. Endoscopy of the gastroduodenal mucosa after carprofen, meloxicam and ketoprofen administration in dogs. J. Small Anim. Pract. 39: 421-424.

10. Forsyth, S. F., Guilford, W. G. and Lawoko, C. R. O. 1996. Endoscopic evaluation of the gastroduodenal mucosa following non-steroidal anti-inflammatory drug administration in the dog. N. Z. Vet. J. 44: 179-181.

11. Giles, A. R., Tinlin, S. and Greenwood, R. 1982. A canine model of hemophilic(factor CIII:C deficiency) bleeding. Blood 60: $727-730$.

12. Grauer, G. F., Greco, E. S., Behrend, E. N., Mani, I., Fettman, M. J. and Allen, T. A. 1995. Estimation of quantitative enzymuria in dogs with gentamicin-induced nephrotoxicosis using urine enzyme/creatinine ratios from spot urine samples. J. Vet. Intern. Med. 9: 324-327.

13. Grisneaux, E., Pibarot, P., Dupuis, J. and Blais, D. 1999. Comparison of ketoprofen and carprofen administration prior to orthopedic surgery for control of postoperative pain in dogs. $J$. Am. Vet. Med. Assoc. 215: 1105-1110.

14. Heiene, R., Moe, L. and Molmen, G. 2001. Calculation of urinary enzyme excretion, with renal structure and function in dogs with pyometra. Res. Vet. Sci. 70: 129-137.

15. Hennan, J. K., Huang, J., Barrett, T. D., Driscoll, E. M., Willens, D. E., Park, A. M., Crofford, L. J. and Lucchesi, E. R. 2001. Effects of selective cyclooxygenase-2 inhibition on vascular responses and thrombosis in canine coronary arteries. Circulation 104: 820-825.

16. Husdan, H. and Rapoport, A. 1968. Estimation of creatinine by the Jaffe reaction. Clin. Chem. 14: 222-238.

17. Jergens, A. E., Turrentine, M. A., Kraus, K. H. and Johnson, G. S. 1987. Buccal mucosa bleeding times of healthy dogs and of dogs in various pathologic states, including thrombocytopenia, uremia, and von WillebrandÅfs disease. Am. J. Vet. Res. 48: $1337-1342$.

18. Jonston, S. A. and Budsberg, S. C. 1997. Nonsteroidal antiinflammatory drugs and corticosteroids for the management of canine osteoarthritis. Osteoarthritis 27: 841-862.

19. Khan, K. N. M., Venturini, C. M., Bunch, R. T., Brassard, J. A., Koki, A. T., Morris, D. L., Trump, B. F., Maziasz, T. J. and Alden, C. L. 1998. Interspecies differences in renal localization of cyclooxygenase isoforms: implications in nonsteroidal antiinflammatory drug-related nephrotoxicity. Toxicol. Pathol. 26: 612-620.

20. Lawson, D. D. 1971. Degenerative joint disease. The treatment of canine degenerative joint disease. J. Small Anim. Pract. 12: 101-103.

21. Lewis, J. H. 1984. Hepatic toxicity of nonsteroidal anti-inflammatory drugs. Clin. Pharm. 3: 128-138.
22. Lobetti, R. G. and Joubert, K. E. 2000. Effect of administration of nonsteroidal anti-inflammatory drugs before surgery on renal function in clinically normal dogs. Am. J. Vet. Res. 61: 1501-1507.

23. MacPhail, C. M., Lappin, M. R., Meyer, D. J., Smith, S. G., Webster, C. R. L. and Armstrong, P. J. 1998. Hepatocellular toxicosis associated with administration of carprofen in 21 dogs. J. Am. Vet. Med. Assoc. 212: 1895-1901.

24. McCafferty, D., Granger, D. N. and Wallace, J. L. 1995. Indomethacin-induced gastric injury and leukocyte adherence in arthritic versus healthy rats. Gastroenterology 109: 11731180.

25. McNeil, P. E. 1992. Acute tubulo-interstitial nephritis in a dog after halothane anaesthesia and administration of flunixin meglumine and trimethoprim-sulphadiazine. Vet. Rec. 131: $148-151$.

26. Numa, H. 1987. Study of $\gamma$-GTP activity in urine and renal tissue of experimental warm ischemic kidney. Hinyokika. Kiyo. 33: $1562-1568$.

27. Rodriguez, F., Llinas, M. T., Gonzalez, J. D., Rivera, J. and Salazar, F. J. 2000. Renal changes induced by a cyclooxygenase-2 inhibitor during normal and low sodium intake. Hypertension 36: 276-281.

28. Sato, R., Soeta, S., Miyazaki, M., Syuto, B., Sato, J., Miyake, Y., Yasuda, J., Okada, K. and Naito, Y. 2002. Clinical availability of urinary $\mathrm{n}$-acetyl- $\beta$-d-glucosaminidase index in dogs with urinary diseases. J. Vet. Med. Sci. 64: 361-365.

29. Sato, R., Soeta, S., Syuto, B., Yamagishi, N., Sato, J. and Naito, Y. 2002. Urinary excretion of n-acetyl- $\beta$-d-glucosaminidase and its isoenzymes in cats with urinary disease. J. Vet. Med. Sci. 64: 367-371.

30. Seibert, K., Zhang, Y., Leahy, K., Hauser, S., Masferrer, J. and Isakson, P. 1997. Distribution of COX-1 and COX-2 in normal and inflamed tissues. Adv. Exp. Med. Biol. 400A: 167-170.

31. Slingsby, L. S. and Waterman-Pearson, A. E. 2000. Postoperative analgesia in the cat after ovariohysterectomy by use of carprofen, ketoprofen, meloxicam or tolfenamic acid. J. Small Anim. Pract. 41: 447-450.

32. Uechi, M., Wakao, Y. and Takahashi, M. 2002. Evaluation of the progress of drug-induced renal disorder by using urinary enzyme in rats. J. Anim. Clin. Med. 10: 179-184.

33. Uechi, M., Nogami, Y., Terui, H., Nakayama, T., Ishikawa, R., Wakao, Y. and Takahashi, M. 1993. Evaluation of urinary enzymes in dogs with early renal disorder. J. Vet. Med. Sci. 56: 555-556.

34. Venturini, C. M., Isakson, P. and Needleman, P. 1998. Nonsteroidal anti-inflammatory drug-induced renal failure: a brief review of the role of cyclo-oxygenase isoforms. Curr. Opin. Nephrol. Hypertens. 7: 79-82.

35. Vonderhaar, M. A. and Salisbury, S. K. 1993. Gastroduodenal ulceration associated with flunixin meglumine administration in three dogs. Am. J. Vet. Med. Assoc. 203: 92-95.

36. Watson, A. D., Nicholson, A., Churchi, D. B. and Pearson, M. R. 1996. Use of anti-inflammatory and analgesic drugs in dogs and cats. Aust. Vet. J. 74: 203-210. 